\title{
Sensibilität und Motivation zum sozialen Handeln in der Auffassung Karol Wojtylas
}

\author{
Sensitivity and motivation for social action \\ in Karol Wojtyla's thought
}

\begin{abstract}
Sensitivity, as the ability to respond to internal and external stimuli, undoubtedly plays an important role in the social action of persons. Without this ability -as in the case of indifference- human being lacks a perception that is necessary in response to a specific emergency situation or a need. This article focuses on the teaching of Karol Wojtyla in his major work Acting Person, with a particular emphasis on the question of the motivation of human action. We want to reply to three basic questions related to the topic: Does this ability strengthens or weakens the culture of the Internet age, especially in Western European society? What is the primary motive of human action? Is sensitivity an essential motive and a factor of development of the person and society? What practical consequences we can draw from the teachings of the Krakow thinker about sensitivity? A person, as defined by Wojtyla, that is isolated, remains indifferent. Similarly persons who are hypersensitive hinders themselves to a mature participation in responsible love. The first quality (indifference) leads persons to flee from their duties. The second (hypersensitivity) leads persons to be overwhelmed by this challenge. The first we can describe as lack of feeling, the second as excessive compassion. The diagnosis of Wojtyla (it is more important to be than to have) remains very current and is an important stimulus in which direction to go to promote motivation for social action, and to build a society of communion of persons. Instead of an individualistic dominated competitive struggle of egos, we should try to realize a society in which the encounter of persons is characterized by mutual devotion in love.
\end{abstract}




\section{Keywords}

Sensitivity, Karol Wojtyla, Acting Person, action of man, social action.

Die Sensibilität als Fähigkeit auf interne und externe Impulse eine Antwort zu geben spielt zweifelsohne eine wichtige Rolle im sozialen Handeln des Menschen. Ohne diese Fähigkeit -wie im Falle der Gleichgültigkeit - fehlt dem Menschen eine Wahrnehmung, die notwendig ist, um eine Antwort auf eine konkrete Notsituation, ein Bedürfnis zu geben. Ein gleichgültiger Mensch ist, ähnlich wie ein Blinder oder Tauber, nicht fähig die entsprechenden Zeichen und Signale zu sehen oder zu hören. Gleichzeitig müssen wir schon am Anfang unserer Überlegungen betonen, dass genauso wie es eine Unempfindlichkeit (Gleichgültigkeit)gibt, es auch ein anderes Extrem geben kann, das für soziales Handeln mit Schwierigkeiten verbunden ist, nämlich die Überempfindlichkeit. In der Frage nach der Motivation des menschlichen Handelns wollen wir uns im vorliegenden Artikel auf die Lehre Karol Wojtylas in seiner vorpäpstlichen Periode konzentrieren. ${ }^{1}$ Wir wollen auf drei Grundfragen, die mit dem Thema zusammenhängen, antworten:

1. Stärkt oder schwächt die Kultur des Internetzeitalters, besonders in unserem westeuropäischen Kreis, die Fähigkeit zu sozialem Handeln?

2. Was ist das primäre Motiv des menschlichen Handelns? Hat Kant Recht, wenn er die autonome Vernunft als Entscheidungsträger nach dem kategorischen Imperativ als Voraussetzung für menschliches Handeln betont, oder müssen wir eher Max Scheler Recht geben und die Gefühle (oft unbewusst) der menschlichen Person und den Menschen als ein animal emotionale anerkennen und seine Handlungen primär unter dem Einfluss der Gefühle und Empfindlichkeiten stehend interpretieren? In welche Richtung geht Wojtylas Denken? Was sagt er zur Sensibilität in der Relation Ich-Du und ihre Bedeutung für das soziale Handeln?

3. Ist die Sensibilität ein wesentliches Motiv und ein Entwicklungsfaktor der Person und Gesellschaft? Welche praktischen Konsequenzen können wir aus der Lehre des Krakauer Denkers über die Sensibilität ziehen?

${ }^{1}$ Vor allem konzentrieren wir ans auf das Hauptwerk Karol Wojtylas, Person und Tat. Schon im ersten Satz des Werkes Person und Tat lesen wir: „Studium niniejsze powstaje $\mathrm{z}$ potrzeby obiektywizacji w dziedzinie tego wielkiego procesu poznawczego, który można określić jako doświadczenie człowieka”. K. Wojtyła, Osoba i czyn, Kraków 1969, S. 5. 


\section{Toleranz und Relativierung der Werte als Merkmale unserer gegenwärtigen Kultur}

Die liberalistische Auffassung vom Menschen überbetont die individuellen Freiheiten des Einzelnen bei gleichzeitiger Marginalisierung der Werte wie das Gemeinwohl und der Pflichten, die mit den Freiheitsrechten zusammenhängen. Aufgrund der Relativierung der Werte wird eine moralisch neutrale, multikulturelle Lebensauffassung propagiert. Egoismus und Gier werden infolge der Ökonomisierung des gesellschaftlichen Lebens zu Tugenden hochstilisiert. Der Mensch, vor allem in den wohlhabenden westlichen Kulturkreisen, wird verstanden als Individualist, ohne soziale Verpflichtungen, was sogar dazu führt, dass man ernsthaft von „,nutzlosen überzähligen Menschen“ spricht. Ein typischer Vertreter dieser Denkart, Sigmund Baumann, bezeichnet die Hälfte der Menschheit als „Abfall“. ${ }^{2}$ Wenn wir dazu noch die Theorie des Sozialdarwinismus heranziehen, in der die Natur gemäß ihrem Gesetz determiniert: der Stärkere gewinnt, dann sehen wir bereits deutlich eine Rechtfertigung für die Totalitarismen, die sich auch unter dem Deckmantel der Demokratie und der Mediengesellschaft verbergen können. Da wird subtil und raffiniert dem Menschen seine subjektive Kraft und Würde genommen - seine autonome Selbstbestimmung - was dazu führt, dass der Mensch auf die gleiche Größe wie alle anderen Lebewesen reduziert wird. In ihrem Kern ist diese Auffassung rein materialistisch und positivistisch gegen die außergewöhnliche Stellung des Menschen in der Welt gerichtet. Es wird nicht nur die religiöse Auffassung der Sonderstellung des Menschen torpediert, sondern auch jene philosophische Affirmation, die auf die Würde der Person und ihre Unantastbarkeit hinweist, unterhöhlt und negiert. Diese materialistische Auffassung des Menschen ist nicht nur für die linke und kommunistische Denkart typisch, auch die liberale und ökonomistische Lehre ist dieser Perspektive sehr verwandt. Beide sind, wie schon Wilhelm Ketteler

2 „Zbędność zakłada trwałość i naturalność takiego stanu. (...) Być zbędnym znaczy być nadliczbowym, niepotrzebnym, bezużytecznym - niezależnie od tego, jaki rodzaj potrzeb i użytków określa standard użyteczności i nieodzowności. Inni cię nie potrzebują, poradzą sobie równie dobrze - a nawet lepiej - bez ciebie. (...) Uznać kogoś za zbędnego znaczy wyrzucić go dlatego, że przeznaczony jest do wyrzucenia. (...) Zbędność dzieli swe pole znaczeniowe z takimi słowami jak »odrzuty«, »wyrzutki«, »śmieci« - krótko mówiąc odpady”. Z. Bauman, Życie na przemiał, Kraków 2004, S. 25. Sieheauch: U. Beck und J. Willms, Freiheit oder Kapitalismus, Frankfurt am Main 2000, S. 137. 
gesagt hat, Geistesbrüder. Es ist deshalb kein Wunder, dass dank der modernen technischen Möglichkeiten im Globalisierungszeitalter diese de facto totalitäre Ideologie ihre Macht soweit entfalten und festigen konnte.

Die zahlreichen Krisen unserer westlichen Gesellschaft (Finanz-, Vertrauens-, Umwelt-, Glaubens,- und Sinnkrisen) gehen auf diese inneren Angriffe gegen das Menschenbild, das den Menschen seiner Fähigkeit zur Transzendenz beraubt, zurück. Der Mensch wurde „befreit“ von konstanten, unveränderbaren Prinzipien und Normen, die mit den religiösen, übernatürlichen Wahrheiten verbunden sind. Gerade durch die Ausklammerung der Religion und der Gottesfrage (Nietzsche) aus der Öffentlichkeit wird nicht nur äußerlich Neutralität und Multikulturalität verkündet, sondern de facto die Verwirrung der Werte, ihre Relativierung und Subjektivierung erreicht. Der Einzelne wird damit allein gelassen, ihm wird Freiheit suggeriert, er soll sich seine Werte selber aussuchen und sein eigenes, ihm passendes Lebensprogramm basteln, ohne Anspruch auf eine dauerhafte, objektive Gültigkeit dieses Programms. Im Gegenteil, ein Versuch über die objektive Wahrheit zu sprechen und gewisse Normen aus der Macht der mehrheitlichen Entscheidungskompetenzen der Parlamente und anderer Gremien herauszunehmen, wird sofort mit dem Verdacht des Fundamentalismus belegt. Diese Situation führt dazu, dass man in der Öffentlichkeit nicht nur die Religion verbannt, sondern auch alle Auffassungen vom Menschen, die aus rein philosophischer Logik für die Würde der Person argumentieren und die Menschenrechte verteidigen, als verdächtig, vor allem in den Massenmedien, betrachtet. Propagiert wird Toleranz. Dieses Wort wird fast sakralisiert, wehe dem, der sich gegen das Toleranzprinzip stellt. Eine Äußerung, die die Wertehierarchie und ihre objektive Wahrheit betont wird gerne in der Öffentlichkeit als Relikt der Vergangenheit (Mittelalter), abgestempelt. Ein Verstoß gegen das Toleranzprinzip wird mit höchsten Strafen belegt und als Kardinalssünde betrachtet. Den letzten Unsinn müssen alle genauso respektieren und dulden wie die größte Schönheit und Wahrheit. Alle müssen die unbegrenzte Freiheit eines Künstlers, Journalisten oder eines Internetbloggers tolerieren. Es gehört wie selbstverständlich zum Zeitgeist, dass z.B. die Religion (vor allem die christliche und muslimische) auf alle mögliche Art und Weise beschimpft und beleidigt werden kann und darf. Gerne wird dann die Freiheit des Autors solcher Programme oder Zeitungen, Filme usw. als Hauptmerkmal unserer europäischen Kultur hervorgehoben. Obwohl es keine Rechtfertigung für Mord und Gewalt gibt, kann das Beispiel 
der französischen Zeitung "Charlie Hebdo“ uns hier als Beleg dienen. ${ }^{3}$ Das Recht der Journalisten den muslimischen Glauben zu beleidigen wurde als selbstverständlich empfunden. Niemand versuchte auch nur ansatzweise die Gründe für die unglaubliche Wut und Barbarei der Täter zu verstehen. Noch einmal muss betont werden, dass der Anschlag aufs schärfste zu verurteilen ist, jedoch hat die schnelle und weltweite Solidaritätsbekundung mit den Opfern eine wirkliche Aufarbeitung der Problematik bis heute nicht bewirkt. Auch ein Verhöhnen des Christentums (blasphemische Darstellung des Gekreuzigten,...) wird als Freiheit der Kunst gebilligt und legalisiert. Wenn sich Christen oder Muslime beleidigt fühlen und dagegen protestieren, werden sie in den Medien als intolerant abgestempelt. Sie sollen die Andersdenkenden tolerieren. Dass eine asymmetrische und ungerechte Behandlung der großen Mehrheiten durch einflussreiche Minderheiten geschieht, wird oft kommentarlos verschwiegen.

Soziologen betonen einen Zuwachs der Unsicherheit. ${ }^{4}$ Dies lässt sich leicht mit dem oben Gesagten verbinden. Wenn alles toleriert werden soll, dann gibt es in der Öffentlichkeit Raum für eine Kultur des Atheismus, Satanismus, der Gewalt, des Konsumismus und des Todes, die den Menschen, dem Einzelnen, sowie den Gesellschaften Orientierungslosigkeit, viele Turbulenzen, Risiken und Unsicherheiten beschert. Wir beobachten bereits die Zunahme der Depressionen, der Selbstmorde, wachsende Akzeptanz für Euthanasie und Verlust des Lebenssinns, was mit dem Menschenbild zusammenhängt. Es lohnt sich einfach nicht mehr zu leben, außer man ist jung, fit, schön und reich.

Unsere Kultur schwächt damit unsere wahre Sensibilität, das Vertrauen und echte Solidarität. ${ }^{5}$ Wenn wir mit Konsum, einer „neuen Kunst“, Gewaltvideos, der Mode der Mainstream konformen „Wahrheiten“ umworben werden, müssen wir uns als logische Konsequenz in die Gleichgültigkeit oder

3 "Attacks like the one on "Charlie Hebdo" aim to destroy freedom of expression and tolerant societies. But we must not and shall not give up our rights to freedom of opinion and freedom of the press. This is why we firmly and continuously uphold free speech and freedom to publish all over the world. Transparency and education are today more important than ever. We must ensure that such attacks do not provide an occasion for dangerous polarization". http://wirsindcharlie. de/ (21.01.2015).

${ }^{4}$ U. Beck, Vom Verschwinden der Solidarität, Berlin 1993, S. 39n. S. Beck, Risikogesellschaft. Risikogesellschaft. Auf dem Weg in eine andere Moderne, Frankfurt am Main 1986.

${ }^{5}$ Z. Waleszczuk, Solidarność w nauczaniu Jana Pawła II (Solidariät in der Lehre Johannes Pauls II.), Kraków 2014. 
Überempfindlichkeit zurückziehen. Beides führt zu einer Starre, einer inneren Unfähigkeit auf die Impulse (von außen oder von innen) adäquat und reif zu reagieren.

Zusammenfassend können wir am Beispiel der Ereignisse um die Zeitschrift Charlie Hebdo folgendes festhalten: eine kleine Minderheit (8 Personen) nimmt sich das Recht eine große Mehrheit (1,1 Milliarden Muslime) zu beleidigen und beruft sich dabei auf ihr absolut verstandenes Recht auf freie Meinungsäußerung. Dass dabei die Religionsfreiheit angegriffen wird, wird stillschweigend akzeptiert, dass dabei rein arithmetisch gegen das Demokratieprinzip verstoßen wird, wird ebenfalls verschwiegen. Statt dessen wird von den Medien und den großen Politikern der EU zu Solidaritätsbekundungen und Massenversammlungen aufgerufen und ein Aufschrei der Empörung artikuliert, dass die gläubigen Muslime die Provokateure aus Frankreich auf so eine unverständliche und menschenunwürdige Art behandelt haben, dass es keine Erklärung und keine Rechtfertigung für diese Taten gibt. Diese Terroristen haben gegen die Heiligkeit des Toleranzgesetzes verstoßen, deshalb werden sie zu Monstern stilisiert und so verhindert, dass über die wahren, tieferen Motive, die hinter diesen Taten stecken, nachgedacht wird. Im Gegenteil wird oft jedes selbständige Denken durch die Massenmedien und die Handlungen der Politiker verhindert. An diesem Beispiel können wir sehen wie der gegenwärtigen linksliberalen Kultur die wahre Sensibilität für andere Kulturen und Denkarten verschlossen bleibt. Wie sie in Wirklichkeit diktatorisch vorgeht und nur eine erlaubte Denkart zulässt, wie sie auch letztendlich gegen die Demokratieprinzipien verstößt und anstatt die Argumente der wahren Mehrheit zu präsentieren, nimmt sie die Seite einer kleinen Minderheit. Alle Zuschauer und Empfänger solcher Nachrichten sollen sich damit ausschließlich und automatisch mit den Satirikern solidarisch erklären. Die Empfindlichkeit und ein Verständnis für die religiös motivierte Empörung werden quasi verboten. Am dargestellten Beispiel können wir symptomatisch betrachten, wie die moderne Kultur den einfachen Bürger und Konsumenten in seiner Sensibilität behindert und einseitig manipuliert. Er soll soweit tolerant sein, dass er alle Angriffe gegen seine eigene oder andere Glaubensüberzeugungen regungslos duldet. Dies führt selbstverständlich zu einer Gleichgültigkeit oder schlägt um in das Gegenteil der Überempfindlichkeit. Beides erlahmt die Motivation zu adäquaten solidarischen Handlungen. 


\section{Der Mensch zwischen Selbstbestimmung und Unfreiheit (Gleichgültigkeit, Überempfindlichkeit)}

In der Sozialdiagnose Wojtylas erfährt jeder Mensch eine moralische Empfehlung das Gute zu erfüllen. Er steht im Drama der Entscheidung zwischen zwei Welten zu wählen: einerseits die Liebe, das Gute und das Leben, andererseits den Hass, das Böse und den Tod. Nach Wojtyla dominiert heute, besonders in unserem westlichen Kulturkreis eine Welt, die Schwäche, die Vergänglichkeit der Existenz des Menschen und ihren Wert relativiert und unterminiert, somit eine Zivilisation des Todes. ${ }^{6}$ Der Schlüsselbegriff mit dem Wojtyla in seinem Denken diese negative Entwicklung des westlichen Menschen beschreibt lautet Alienation. Für unser Thema können wir diesen Begriff auch mit Gleichgültigkeit wiedergeben. Die Gleichgültigkeit (Alienation) steht nach Wojtyla in Opposition zur wahren Antwort auf die Herausforderungen des Menschen d.h. die Teilnahme, was für unser Thema als Sensibilität zu beschreiben wäre. Selbstverständlich greift der Begriff der Teilnahme tiefer und die Sensibilität ist wie eine Vorstufe ein Teil dieser Haltung. ${ }^{7}$ Sensibilität betont mehr die emotionale Fähigkeit auf die Impulse von außen oder von innen zu reagieren, was selbstverständlich für den Autor von Person und Tat von Bedeutung ist. Man muss jedoch auch die Rolle der freien Entscheidung, den freien Willen betonen und berücksichtigen. Bezugnehmend auf die bei der Einführung gestellte Frage nach dem Motiv des menschlichen Handelns, ob nun Max Schelers Auffassung von der primären Bedeutung der Gefühle oder Immanuel Kant, der die Vernunft (vor allem die praktische, den in den moralischen Entscheidungen sich äußernden Willen)als Ausschlag gebend betont, spricht sich Wojtyla eindeutig für die zweite Antwort aus. Im Gegensatz zu vielen Interpretatoren, die Wojtylas Denken unbedingt als Thomismus, oder als Phänomenologie darzustellen versuchten, wird hier im Ansatz des Krakauer Philosophen die Freiheit des Willens und die Erfahrung des kategorischen Imperativs, bei jedem Menschen (die damit die Personenwürde im moralischen Gesetz offenbart) als dass das wichtigste Motiv hervorgehoben.

Für unsere Überlegungen ist es von Bedeutung, das Wojtyla die Würde des Menschen, die sich in der Tat, vor allem in der Relation meines „ich“

${ }^{6}$ Sollocitudo Rei Socialis 36.

7 Z. Waleszczuk, Wolność osoby ludzkiej w ujęciu Karola Wojtyły i Immanuela Kanta (Freiheit der menschlichen Person bei Karol Wojtyla und Immanuel Kant), Wrocław (Breslau) 2014. 
gegenüber dem anderen „Du“ offenbart, ${ }^{8}$ als ein Personenmerkmal beschreibt und gleichzeitig neben der Betonung der Willensfreiheit und der Fähigkeit zur Selbstbestimmung der handelnden Person (in der Tat) auch eine zweite Eigenart des Menschen betont, die Fähigkeit zur Liebe. „Nur Personen haben an der Liebe teil “. ${ }^{9}$ Diese Fähigkeit an der Liebe teilzunehmen ist mit unserem Hauptproblem verbunden, das wir als Sensibilität beschreiben können. Eine Person, so können wir im Sinne Wojtylas sagen, die sich isoliert, d.h. gleichgültig bleibt, ebenso auch eine Person die überempfindlich ist erschwert sich damit eine reife Teilnahme an der verantwortungsvollen Liebe. Durch die erste Eigenschaft (Gleichgültigkeit) wird eine solche Person vor ihren Aufgaben fliehen, durch die zweite (Überempfindlichkeit) wird sie von dieser Herausforderung erdrückt. Das erste können wir als Gefühllosigkeit beschreiben, das zweite als übertriebenes Mitleiden. Die Gleichgültigkeit können wir mit Blindheit oder Taubheit vergleichen, der Mensch kann die entsprechenden Impulse nicht wahrnehmen und verbleibt in Wojtylas Sprache in Alienation. Eine andere Form, die aber ähnlich endet, ist die Überempfindlichkeit, ${ }^{10}$ sie belastet und beschäftigt die Person so stark mit Gefühlen, dass sie eine freie, nüchterne und vernünftige Entscheidung unmöglich macht, der Mensch wird dann durch die Gefühle gesteuert und reagiert überschnell und unüberlegt. Eine solche launenhafte Haltung erreicht nie den Status einer reifen, verantwortlichen Liebe, es fehlt die reife Tat als freier und selbstbestimmender Wille der handelnden Person.

Gleichgültigkeit bedeutet eine fehlende Zusammenarbeit, Wojtyla benutzt hier das Wort Alienation, anknüpfend an die damals herrschende marxistische Philosophie. Nicht nur der Marxismus, so würde der Autor von Person und Tat heute sagen, führt zu Alienation, d.h. Gleichgültigkeit und fehlender Bereitschaft zur Zusammenarbeit zwischen den Personen, sondern auch der liberale Individualismus atomisiert und, wie wir oben aufgezeigt haben, birgt die gleichen Gefahren in sich. Der Autor von Person und Tat hat Recht, wenn er betont, dass „ehrliches Verständnis des menschlichen Handelns kann zu

8 Z. Waleszczuk, Die Person bei Immanuel Kant und Karol Wojtyla, Breslau 2014.

9 K. Wojtyła, Osoba i czyn, S. 131.

${ }^{10}$ Diese Überlegungen lassen sich nicht expressis verbis aus Person und Tat ablesen, sie sind jedoch implicite vorhanden. Besonders in der Beschreibung der Bedeutung der Autonomieentscheidung der Freiheit des Willens in der Ich-Du-Beziehung kann man diese Gedanken finden. 
richtiger Interpretation der Zusammenarbeit" führen. ${ }^{11}$ Bezugnehmend auf das Thema unserer Untersuchung können wir also auf die bedeutende Rolle der Sensibilität hinweisen, als Wunsch nach Zusammenarbeit und Motiv des sozialen Handelns. Wojtyla stellt in seinem Hauptwerk eine wichtige Frage, die immer noch aktuell geblieben ist und sogar an Bedeutung gewonnen hat: „es stellt sich die Frage, ob unsere Zivilisation, besonders die atlantische mit ihrem Primat von Eigennutz und Technik, in der Gefahr der Alienation steht“. ${ }^{\text {"2 }}$ Unter dem Begriff Alienation können wir auch Gleichgültigkeit als Merkmal der fehlenden solidarischen Haltung, als Mangel an Sensibilität für die Not der anderen verstehen. Auch in unserer heutigen Gegenwart erleben wir, wie der erste Teil unserer Untersuchung skizzenhaft darlegt, durch die zunehmenden Krisen in vielen Bereichen, viele Situationen des Betrugs, der Ausbeutung, der Falschheit, nicht nur im individuellen, sondern auch in institutionellen und strukturellen Bereichen. Ein unsoziales Verhalten, das sich vor dem Gemeinwohl verschließt und sich nur an möglichst schnellen und großen Profiten orientiert, gehört zum typischen Erscheinungsbild unserer globalisierten Welt. Die Banken, die Medien, auch die großen Konzerne handeln oft nur zum schnellen Vorteil der Stärkeren, ${ }^{13}$ was als Folge aber auch demoralisierend auf die ganze Gesellschaft wirkt. Wojtyla sagte, der Täter wirkt nicht nur auf das andere Du, sondern auch auf sich selbst durch seine Tat. Wenn ein Bankier oder Manager seine Kunden absichtlich ausnutzt, falsch informiert oder betrügt, dann befindet er sich, in der Sprache Wojtylas, in einer Gleichgültigkeitshaltung, er bleibt unsensibel für die Forderung des moralischen Gesetzes. Er bleibt gleichgültig für die Forderung der personalistischen Norm, die in sein Herz eingeschrieben wurde. Die Stimme des eigenen Gewissens kann abstumpfen und schließlich taub werden lassen, keine moralischen Bedenken mehr haben und sich selbst so hart verschließen, dass man unempfindlich bleibt. Dies aber, so Wojtyla, führt zu einem Absterben meines eigenen Ich, denn dadurch entfalte ich mich nicht,

${ }^{11}$ K. Wojtyla, Osoba i czyn oraz inne studia antropologiczne, Towarzystwo Naukowe KUL, Lublin 2000, S. 303.

${ }^{12}$ K. Wojtyła, Uczestnictwo czy alienacja?, w: K. Wojtyła, Osoba i czyn oraz inne studia antropologiczne, Towarzystwo Naukowe KUL, Lublin 2000, S. 456.

13 „Karę w wysokości 2,5 mld euro zgodziło się zapłacić w listopadzie 2014 r. pięć globalnych banków (...) za manipulacje na rynkach wymiany walut”. Nadzorcy rynków finansowych znaleźli dowody na to, że banki stworzyły kartel ustalając kursy walut: „kurs waluty nie był wypadkową transakcji na rynku, lecz rozmów bankierów w kuluarach”. J. Piński, Przekręt stulecia, Wrocław 2014, S. 7. 
ich verleugne mich selbst. Der Mensch der sich selbst nicht treu bleibt, sprich auf die Stimme des Gewissens nicht mehr hören will -, gerät in eine Lage, die ihn nicht nur gegenüber den anderen Menschen gleichgültig macht, sondern letztlich auch gegenüber sich selbst, er lebt dann nach dem Prinzip nutzen und benutzen, d.h. den anderen als Sache und auch sich selbst als Sache zu betrachten. Dieses egoistische Modell entwürdigt die andere Person, aber auch mich selbst. Die personalistische Norm (kategorischer Imperativ) ${ }^{14}$ verlangt nach der Antwort eines sensiblen Herzens. Wie wir bereits oben aufgezeigt haben, befindet sich unsere gegenwärtige Kultur auf einem Kollisionskurs gegen diese Freiheit des Gewissens. Anstatt die Sensibilität und die damit verbundene reife Entscheidungsfähigkeit der Personen zu fördern, werden die Menschen durch die Propagierung der materialistischen und nihilistischen Ideologien zu Gleichgültigkeit und Unfreiheit erzogen.

\section{Wir sind zur Tat berufen}

Wojtyla erinnert an die Wichtigkeit der Achtung vor der menschlichen Person. Er weist auf die personalistische Norm (kategorischer Imperativ) hin. Die Gefahr, die durch Alienation droht, sieht Wojtyla nicht nur im ökonomischen, politischen und kulturellen Bereich. Auch die Religion wird gemäß dem marxistischen Prinzip, heftig angegriffen. Der Traum von Karl Marx, von einem Ende der Alienation und dem „Beginn eines Königtums der Freiheit und voller Befreiung für alle", wurde noch nicht ausgeträumt, obwohl der Kommunismus 1989 politisch in Europa zu Ende ging. Die westeuropäischen Kulturkreise unserer linksliberalen Eliten haben sich immer noch nicht von ihrem Stiefvater Marx getrennt. Die Freiheitsvorstellungen und die Machtverhältnisse im gegenwärtigen Europa führen uns zu der nüchternen Bemerkung, dass wir an der Spitze der Politik, Kultur und Wirtschaft nur eine dominierende Linie, nämlich die überdimensional vertretenen linken Herrscher haben. Aus Brüssel kommen heute eindeutige kulturelle Direktiven, die uns allen eine neue Ordnung und ein Menschenbild der Toleranz als das wichtigste Prinzip vorschreiben. Nicht die Wahrheit, sondern Multikulturalität soll unsere Ordnung bestimmen.

${ }^{14}$ Z. Waleszczuk, Der Kategorische Imperativ als Grundstein der Menschenwürde und Form der Offenbarung Gottes bei Immanuel Kant und Karol Wojtyla, "The Person and the Challenges" 3 (2013) Nr 1, S. 7-42. 
Diese einseitig verstandene Freiheit, die eigentlich unsere Solidaritätsbereitschaft reduziert, ${ }^{15}$ den Menschen verunsichert und orientierungslos macht, braucht eine Korrektur. Wir können mit Wojtyla daran erinnern, dass der Mensch zu Hingabe und Erfüllung berufen ist, die als communio personarum zu verstehen ist. Erst die Wiederentdeckung der tieferen Wurzeln der menschlichen Person, die auf Transzendenz hinweisen, führt uns, so Wojtyla, zu der wahren Freiheit, die in einer verantwortliche Liebe mündet. Der Mensch der sich gleichgültig isoliert, kann seine existentielle Erfüllung nicht finden, er bleibt verschlossen und ist nicht er selbst. Nur dann, wenn der Andere als ein „Du“ gegenüber meinem „Ich“, als ebenso würdig verstanden wird, wenn ich ihn behandle, wie ich selbst behandelt werden will, d.h. wenn ich sensibel bin und die Empathie habe den anderen so wahrzunehmen wie er ist, mit all seinen Nöten, Freuden, Sorgen, Ängsten und Hoffnungen, erst dann verwirklicht sich meine Menschlichkeit. Wenn der andere nur als Fremder, den ich zu nutzen weiß, betrachtet und behandelt wird, verhindert dies die wahre Entwicklung der Personen. Erst wenn ich die andere Person achte und nicht wie eine Sache instrumentalisiere, sondern versuche für ihn da zu sein und das Gute für ihn zu tun, verwirkliche ich nicht nur seine Bedürfnisse, sondern erfülle das Bedürfnis nach Liebe auch meinerseits, erst dann kommt eine Gemeinschaft zustande, realisiert sich Freundschaft, die Wojtyla als Teilhabe und Wir-Relation beschreibt. Es ist eine Erfahrung des anderen Ich als Begegnung.

\section{Was kann uns befreien?}

Der Autor von Person und Tat erinnert: der Marxismus ist keine geeignete Arznei für den gegenwärtigen Menschen, auch der Individualismus und Materialismus befreien den Menschen nicht. Im Gegenteil sie führen uns zu mehr Gleichgültigkeit und Egoismus, das Los der anderen wird uns immer mehr egal. Bereits die kleinen Kinder werden zu Konkurrenzkampf und Egoismus erzogen. Wenn Eltern keine Zeit mehr finden für eine Begegnung in Liebe, wenn das kleine Kind schon früh vor den Computer abgeschoben und allein gelassen wird, oder in eine Ganztageseinrichtung abgegeben wird, wird der Mensch in seiner Liebeserfahrung gestört. Welche Zukunft hat die Menschheit, wenn wir

15 Siehe: Z. Waleszczuk, Solidarität nach Johannes Paul II, Bayreuth 2004. 
keine Motivation für Empathie, für die Stärkung der Sensibilität und Solidarität, wenn wir sinnstiftenden Grundlagen nicht mehr schützen. ${ }^{16}$

\section{Fazit}

Das moralische Übel steht im Gegensatz zur Erfüllung. Nur die moralische Norm, so Wojtyla, kann uns helfen in der Verwirklichung der Liebe zu handeln. ${ }^{17}$ Die Sensibilität, die die gegenwärtige Kultur und besonders die modernen Medien so häufig schwächen durch die Propagierung von Konsum, Nihilismus und Materialismus, kann sicherlich nicht gestärkt werden durch mehr informationelle Verwirrung, sondern durch eine Kultur des Verzichtes, der Bildung, der Stärkung der Familie und der Erziehung zu selbständiger verantwortlicher Tat.

Selbstverständlich ist unser Thema damit nicht erschöpft, es wurden lediglich einige Signale und Andeutungen gegeben, in welche Richtung wir gehen sollen, wenn wir den gefährlichen Herausforderungen der Gegenwart entgegenwirken wollen. Papst Franziskus sprach im Angesicht der europäischen Krisen in seiner Rede in Straßburg über Europa als eine Oma. Diese betagte Frau ist nicht mehr fruchtbar und beginnt vergesslich zu sein. Sie stirbt aus und an ihre christlichen Wurzeln, ihre Identität erinnert sie sich nicht mehr. Dieses alternde und aussterbende Europa, hat keine Zukunft ohne eigene Empfindlichkeit und ohne seine Erinnerungskraft wieder zu gewinnen. Unseren Ursprung als Gottes Kreatur nicht mehr zu kennen, gleichgültig gegenüber der Stimme des Gewissens zu sein, ist der Ursprung einer falschen Utopie. Laut Wojtyla ist es wichtiger zu sein als zu haben. Die Diagnose Wojtylas bleibt sehr aktuell und ist ein wichtiger Impuls in welche Richtung wir gehen sollen, um eine Gesellschaft der communio der Personen zu bauen. Anstelle eines individualistisch dominierten Konkurrenzkampfes der Egoismen, sollen wir eine Gesellschaft zu verwirklichen versuchen, in der die Begegnung der Personen durch gegenseitige Hingabe in der Liebe geprägt ist.

16 Z. Waleszczuk, Gleichheit der Rechte und Pflichten im Denken Karol Wojtylas, in: W. Zuziak, J. Mysona Byrska (Hrgs.), Równość w życiu publicznym (Gleichheit im gesellschaftlichen Leben), Krakow 2012, S. 315-338.

17 K. Wojtyła, Liebe und Verantwortung. Eine ethische Studie, München 1981. 


\section{Bibliography}

Bauman Z., Życie na przemiał, Kraków 2004.

Beck U., Risikogesellschaft. Risikogesellschaft. Auf dem Weg in eine andere Moderne, Frankfurt am Main 1986.

Beck U., Vom Verschwinden der Solidarität, Berlin 1993.

Beck U. und J. Willms, Freiheit oder Kapitalismus, Frankfurt am Main 2000.

Piński J., Przekręt stulecia, Wrocław 2014.

Waleszczuk Z., Solidarność w nauczaniu Jana Pawła II (Solidariät in der Lehre Johannes Pauls II.), Kraków 2014.

Waleszczuk Z., Wolność osoby ludzkiej w ujęciu Karola Wojtyły i Immanuela Kanta (Freiheit der menschlichen Person bei Karol Wojtyla und Immanuel Kant), Wrocław (Breslau) 2014.

Waleszczuk Z., Solidarität nach Johannes Paul II, Bayreuth 2004.

Waleszczuk Z., Der Kategorische Imperativ als Grundstein der Menschenwürde und Form der Offenbarung Gottes bei Immanuel Kant und Karol Wojtyla, „The Person and the Challenges" 3 (2013) Nr 1, S. 7-42.

Waleszczuk Z., Gleichheit der Rechte und Pflichten im Denken Karol Wojtylas, in: W. Zuziak, J. Mysona Byrska (Hrgs.), Równość w życiu publicznym (Gleichheit im gesellschaftlichen Leben), Krakow 2012, S. 315-338.

Waleszczuk Z., Die Person bei Immanuel Kant und Karol Wojtyla, Breslau 2014.

Wojtyla K., Osoba i czyn oraz inne studia antropologiczne, Towarzystwo Naukowe KUL, Lublin 2000.

Wojtyła K., Liebe und Verantwortung. Eine ethische Studie, München 1981. 